\title{
Neurogenic hypothesis and psychiatric disorders
}

\author{
LAU Benson WuiMan ${ }^{1 *}$, LEE Jada ChiaDi ${ }^{2,3,4} \&$ SO KwokFai ${ }^{2,3,4,5^{*}}$ \\ ${ }^{1}$ Department of Rehabilitation Science, The Hong Kong Polytechnic University, Hong Kong, China; \\ ${ }^{2}$ Department of Anatomy, Li Ka Shing Faculty of Medicine, The University of Hong Kong, Hong Kong, China; \\ ${ }^{3}$ The State Key Laboratory of Brain and Cognitive Sciences, The University of Hong Kong, Hong Kong, China; \\ ${ }^{4}$ Research Centre of Heart, Brain, Hormone and Healthy Aging, Faculty of Medicine, The University of Hong Kong, Hong Kong, China; \\ ${ }^{5}$ GHM Institute of CNS Regeneration, Jinan University, Guangzhou 510632, China
}

Received October 31, 2012; accepted January 9, 2013; published online July 22, 2013

\begin{abstract}
Psychiatric illness, such as affective disorders, anxiety disorders and schizophrenia, exerts exceptional personal burden on affected individuals. Although not physically noticeable, these disorders cost enormously on ones' family and society. Currently pharmaceutical and psychological treatments are generally accepted as effective for psychiatric disorders, while the exact mechanisms underlying the treatment efficacy, etiology and neurobiology of the disorders remain elusive. In the past decade, "neurogenic hypothesis" emerged as an attempt to explain the nature of psychiatric illness. The origination of the hypothesis is based on several pre-clinical and clinical observations. First, stress, which is a common risk factor of the disorders, was found to suppress neurogenesis; second, treatment for the illnesses like antidepressants and antipsychotics were shown to improve neurogenesis and behavioral deficits simultaneously; and third, the therapeutic effect of antidepressants was abolished in animal models when neurogenesis was blocked. Increasing efforts were invested to determine whether neurogenesis is a key to the understanding and treatment of psychiatric disorders, although contrasting results are also found and thus the importance of neurogenesis remains a matter of debate. The present chapter will discuss the recent findings about the involvement of neurogenesis in major depression, anxiety disorders and schizophrenia, and whether neurogenesis would be a potential target for development of the treatment in the future.
\end{abstract}

neurogenesis, psychiatric disorders, major depression, schizophrenia, anxiety disorder, hippocampus, amygdala, subventricular zone

Citation: $\quad$ Lau B W M, Lee J C D, So K F. Neurogenic hypothesis and psychiatric disorders. Chin Sci Bull, 2013, 58: 3188-3198, doi: 10.1007/s11434-0135886-z

According to a national epidemiology study of psychiatric conditions, the 12-month prevalence of psychiatric illnesses among Americans was over 26\% [1], while the most commonly found diagnosis was anxiety disorder $(18 \%)$ and is followed by mood disorders $(9.5 \%)$. Different classes of psychiatric medications including antidepressants, anxiolytic drugs and antipsychotics are available for the treatment of mood, anxiety and schizophrenia disorders, respectively. Although medications have been used for decades, the exact mechanism of their therapeutic actions remains obscure. Furthermore, the psychopathology of the psychiatric ill-

*Corresponding authors (email: hrmaskf@hku.hk; benson.lau@polyu.edu.hk) nesses remains largely elusive. The limited understanding hampers the effectiveness of treatment and, consequently, enormous personal and societal burden are caused due to the diseases. Extraordinary effort was invested in disclosing the disease process, which aims at providing the theoretical basis for the development of more effective treatment methods [2].

One of the brain regions being intensively studied for its involvement in psychiatric illness is the hippocampus [3]. The hippocampus is known for its involvement in learning and memory, cognitive function, mood regulation and responding to stress [4-6]. The function of hippocampus in learning and memory may be attributed to the presence of 
new neurons produced throughout adulthood, namely, adult neurogenesis [7]. Neurogenesis is a process which involves neural progenitor cells production, migration, differentiation, maturation and functional integration into the existing circuit [8]. The discovery of proneurogenic effect of antidepressant [9] elicit the "neurogenesis hypothesis" of depression: due to the importance of hippocampus in mood regulation and memory formation, the symptoms of depressive disorder are due to the decreased production of newborn neurons in hippocampus, and the therapeutic effect of antidepressants requires intact or enhanced neurogenesis [10]. In the past few years this hypothesis has been examined intensively from different perspectives, and current evidence shows that the hypothesis may be partially true: neurogenesis per se is not the sole cause of depressive disorder, while it is likely to be involved in psychiatric symptoms related to depression and the therapeutic effect of antidepressants [10]. Until now the hypothesis has been extended to other psychiatric illness such as schizophrenia and anxiety disorders. Although increasing lines of evidence support the possible involvement of neurogenesis in psychiatric illness, further study is required before conclusion could be drawn. This review discusses recent studies which aimed at exploring the association between neurogenesis and three psychiatric conditions, including depressive disorders, schizophrenia and anxiety disorders. Our goal is to provide a better understanding of the roles of neurogenesis in the pathophysiology and treatment mechanism of the illnesses.

\section{Nurogenesisi: The process}

The term "neurogenesis" is a process which is defined as the ability of the precursor cells to generate functional neurons within the central nervous system (CNS) [11]. This process has been previously believed to occur only during the embryonic development and the early postnatal period, but not in the adult CNS. However, Altman et al. [12] had provided the first evidence showing that new cells in rodents can be generated within the subgranular zone (SGZ) of the dentate gyrus in the hippocampus. Later, a study from Paton et al. [13] had shown that new cells could be generated in the adult canary's forebrain, which has further strengthened the possibility of adult neurogenesis and established the theory that adult neurogenesis occurs primarily in two germinal zones: the subventricular zone (SVZ) of the dentate gyrus (DG) and the subgranular zone (SGZ). Apart from these two neurogenic regions, cell proliferation was reported to occur in other brain regions including neocortex, thalamus, hypothalamus, striatum and septum [14], but whether neurogenesis occurs remains controversial. Furthermore, only SGZ neurogenesis is conclusively demonstrated in the case of human.

The process of adult neurogenesis within the hippocampus and the subventricular zone can be briefly divided into four stages; they are (1) proliferation, (2) migration, (3) differentiation and (4) integration. Activation of the radial glia-like cells which reside in the SGZ within the dentate gyrus of the hippocampus can cause cell proliferation of the progenitor cells and in turn, the generation of the migrating neuroblasts. These cells are able to migrate from the SGZ to the outer granular layer which at this stage, the cells are termed immature neurons. These immature neurons can then be differentiated into dentate granule cells within the hippocampus. At a later stage, the dendrites gradually extend towards the molecular layer of the dentate gyrus with the establishment of synapses which can then integrate to the existing hippocampal-dependent neural circuit [11]. Very similar to the neurogenesis within the dentate gyrus, the activation of the radial glia-like cells which reside in the subventricular zone can cause proliferation of the transient amplifying cells and in turn, cause the generation of the migrating neuroblasts. However, at this stage, rather than migrating a short distance to the granular cell layer within the dentate gyrus, the immature neurons differentiate into neuroblasts within the subventricular zone and are able to migrate in a chain manner via the rostral migratory stream (RMS) pathway to the olfactory bulb. Upon the arrival to the olfactory bulb, maturation of the periglomerular neurons and granule cells will occur with synaptic formation which can be functionally integrated to the neural circuit of the olfactory system [11]. Other than the neuronal lineage, the progenitor cells within these regions can differentiate into glial cells, such as the progenitors derived from the hippocampus have been found to differentiate into oligodendrocytes and astrocytes in cell culture [2]. However, under physiological condition, a large majority of the cells derived from the neurogenic sites become neurons [2].

Kempermann et al. [15] suggested that in order to determine the functional significance of adult neurogenesis, three major components should be considered. They are the cellular level, the network level and also the system level. For example, under physiological condition, the adult neurogenesis within the hippocampus was suggested to be involved in the formation of trace memories [16]. Trace memory is defined as to associate events that occur in different times. In order to determine the functions of adult neurogenesis within the hippocampus, ablation of cells within the neurogenic site is a direct way to investigate its proposed functions. Shors et al. [16] have shown that with the reduction of the new proliferating cells within the dentate gyrus by methyloxymethanol-actate, a DNA methylating agent, the hippocampal trace conditioning task could be impaired. However, with the recovery of the cells production, an improvement of the ability to form trace memories was found. The functional significance of another neurogenic site, subventricular zone, has been currently related to sexual behaviors in rodents. Mak et al. [17] have shown that male pheromone can stimulate neurogenesis within the subventricular zone and the hippocampus of the female 
mice. This leads to the hypothesis that the increase of neurogenesis may be important for female reproductive success mediated by male pheromone. The importance of studying the subventricular zone and olfactory bulb neurogenesis is that olfaction is an important system for the survival of rodents in the wild. In addition, rodents have been shown to highly rely on the pheromone signatures to determine their mates, which acts as a kind of selection in order to give fertile offsprings [17]. The new cells generated from the subventricular zone are able to migrate for a long distance, via the RMS, to the olfactory bulb and differentiate into functional neurons which are highly involved in the olfactory systems. Thus, the new neurons within the olfactory bulb provided from subventricular zone neurogensis is hypothesized to be related to the rodent's sexual behavior. This is further supported by evidence of animal's behavioral test that following corticosterone treatment, cell proliferation in the subventricular zone was suppressed which was correlated with alteration in male rodent's sexual performance [18]. However, with antidepressant treatment using paroxetine, cell proliferation in the subventricular zone, was increased and sexual performance in male rodents was improved [18]. Furthermore, the pro-sexual effect was abolished when neurogenesis was blocked. The examples mentioned above have given the functional significance of adult neurogenesis within the dentate gyrus and the subventricular zone via investigating the changes of the cell number within the neurogenic sites and correlate this change with the animal behavioral tests as the functional readouts.

\section{Neurogenesis and depressive disorder}

Among different psychiatric disorders, depressive disorder is the first one to be associated with altered neurogenesis. Attention was paid to this potential linkage in past decades and this assumption remained controversial. The investigation was initially fueled by findings from several lines of evidence related to the pathophysiology and treatment of depression. First, stress, which is well accepted as a risk factor of depressive disorders and exacerbates mental disorders, was shown to decrease neurogenesis in different animal studies [19]. Animal models of depressive disorders almost ubiquitously showed impaired neurogenesis in terms of both cell proliferation and survival [2]. Second, treatment of depression, such as different classes of antidepressants (e.g. SSRI and agomelatine [20,21]) and exercise, were shown to promote neurogenesis in animal models of depression, Parkinson's disease [22] or non-stressed, healthy animals [9,23]. Third, since the limbic circuit, which includes the hippocampus, is involved in affective behavior [24], new neuron production in the hippocampus was hypothesized to take part in emotional processing. Based on these initial observations, it is proposed that the alteration of neurogenesis in the hippocampus may be the underlying cause of symptoms found in depressive disorders. This association raised the interest in the research community, and the term "neurogenesis hypothesis of depression" was coined to describe the assumption of involvement of new neurons in the disorder [25]. The hypothesis postulates that the pathophysiology of depression is related to decrease in the production of newborn granule cells in the dentate gyrus, while promotion of the new neuron production could reverse the symptoms and is essential for the therapeutic effect of antidepressants [10].

Despite the initial enthusiasm in elucidating the roles of neurogenesis in depression, later studies presented results which queried the assumed direct (or oversimplified) relationship between neurogenesis and depression. A clinical study which used post-mortem brain sections of major depressive patients showed no significant difference in the number of hippocampal proliferating cells between normal controls and depressive patients [26]. However, due to experimental limitation in using human tissues, several aspects should be noted. One of the most important confronting factors is the lack of control on the medication history, including antidepressants, of the patients. Since antidepressant may restore or enhance neurogenesis in the treated patients, this may mask the actual rate of neurogenesis in the depression state. Other aspects worth noting will be discussed later in this chapter. Nevertheless, this was the hallmark study which provided valuable data for human in vivo neurogenesis in depressive disease conditions. In contrast to this study, two recently published studies showed that antidepressant treatment in major depression patients increased the number of neural precursor cells and angiogenesis in the mid and anterior dentate gyrus [27]. The discrepancy between the two studies may be due to the use of different immunochemical markers and quantification methods, which should be verified in future studies. Pre-clinically, suppression of neurogenesis in non-stressed animals did not result in increased depression-like behavior [28,29], which denied the possibility that depressive disorder is caused by down-regulation of hippocampal neurogenesis per se. Thus it is unlikely that the symptoms of depression are caused solely by down-regulation of neurogenesis in human.

Instead of causing depression-like behavior directly, neurogenesis was shown to be required for the therapeutic effect of antidepressants in several animal studies. In a study conducted by Santarelli et al. [30], the consequence of neurogenesis ablation was examined. X-irradiation was employed to specifically block the new neuron proliferation in the hippocampus of mice, which did not affect the cell division of other body parts and systems. This technique decreased the cell proliferation up to $85 \%$ in the control animals. Then the animals were subjected to treatment of antidepressants chronically. With the antidepressant treatment, the sham-operated mice, as expected, showed a decreased latency to enter an anxiogenic area and exhibited a decreased latency in the novelty suppression test. In contrast, mice 
with neurogenesis blocked did not show a decrease in latency in both tasks. As the latency in both tests indicates the level of anxiety, it was concluded that the ablation of neurogenesis prevented the anxiolytic effect of chronic antidepressant treatment. The result is particularly interesting because it showed that intact neurogenesis was required for the antidepressant to be effective. Another study utilized transgenic technology to illustrate the necessity of neurogenesis in rendering the effect of antidepressant [31]. Brainderived neurotrophic factor (BDNF) is a neurotrophin involved in neuronal survival, differentiation and proliferation [32]. Transgenic mice with the receptor for BDNF (receptor tyrosine kinase TrkB) being knocked out showed impaired neurogenesis. The neurogenesis was unable to be restored by antidepressant or exercise, which has been shown to be proneurogenic. Behaviorally, depression- and anxiety-like behavior could not be reduced by the treatments in the transgenic animals. In contrast, when the trkB expression was abolished in differentiated neurons but not neural progenitor cells in the dentate gyrus, the animals showed response to antidepressant treatment with increased neurogenesis and reduced depression-like behavior. The findings were further supported by other studies $[28,33]$ which indicate the lack of antidepressant or anxiolytic effect of psychotropic drugs in the situation of neurogenesis blockage, and a slower recovery of hypothalamic-pituitary-adrenal-axis after stress was found in neurogenesis-deficient mice $[34,35]$. Collectively, although impaired neurogenesis alone did not cause depression-like behavior, it may abolish the therapeutic effect of antidepressants or sustain the state of depressive disorders.

The neurogenesis process is regulated by different external and internal factors. Since the neural progenitors are situated in a microenvironment of complex neural network, neurotransmitter plays a significant role in the regulation process. The neurotransmitter GABA was found to depolarize and promotes the differentiation of NPCs [36]. Serotonin, which is originally associated with depressive disorder, is another neurotransmitter which regulates neurogenesis [37]. Direct stimulation of serotonin receptor 5HT(2B) promotes neurogenesis while inactivation of the receptor abolished the neurogenic and therapeutic effect of SSRI. Although there is lack of evidence to support the expression of NMDA receptors in hippocampal NPCs, glutamate was found to influence hippocampal neurogenesis [38], while the effect of dopamine and acetycholine depends on the type of receptors expressed in the hippocampus [39]. Apart from BDNF, other trophic factors including Vascular endothelial growth factor (VEGF) and insulin-like growth factor 1(IGF1) $[40,41]$ also exhibit effect on neurogenesis. Infusion of both VEGF and IGF1 into the brain decreases depression/anxietylike behavior and increases neurogenesis. Being similar to BDNF, the behavioral and cellular effect of VEGF is likely to be regulated by the cAMP-response element binding protein (CREB) with an elusive mechanism. Increasing lines of evidence suggest that the activation of immune system may alter neurogenesis, although the results are still under debate. NF-kappa B (Nuclear factor kappa B) signaling pathway, which is renowned for its involvement in inflammatory responses, was found to be activated by stress [42] and mediate the stress-induced depression-like behaviors and neurogenesis. Macrophage migration inhibitory factor (MIF, a pro-inflammatory cytokine) is a positive regulator of neurogenesis. MIF knock-out mice suffered from decreased neurogenesis and behavioral defects such as impaired hippocampal dependent memory and increased anxiety-and depression-like behaviors [43]. In contrast, another pro-inflammatory cytokine interleukin 1beta was shown to be elevated in depression patients and decreased the number of newly proliferative cells in the hippocampus [44]. Lipopolysaccharide (LPS) is commonly used to mimic inflammation and it was found that LPS treatment alone did not affect neurogenesis, rather it would abolish the antidepressant effect of fluoxetine, a selective serotonin reuptake inhibitor (SSRI) [42]. From these results, it could be observed that inflammation or immunological responses are involved in the regulation of neurogenesis, while the precise action of each factor needs to be considered individually.

Considering the neurogenesis hypothesis of depression, neurogenesis is altered in depressive disorder and evidence suggested that it is associated with depression. However, it is unlikely that depression is caused by impaired neurogenesis per se. Instead, the reduced neurogenesis may act as a limiting factor for antidepressants or other treatments to exert their effects. Thus neurogenesis may be another target of treatment due to its importance in mediating the effect of antidepressants. The disruption of hippocampal neurogenesis may contribute to symptoms related to hippocampal function like impaired working memory [45], which rationalize targeting neurogenesis when considering treatment for depressive patients. Tang et al. [46] suggested that the complex changes in depression is caused by different intriguing factors, and neurogenesis may be merely one of them. Other factors related to cellular plasticity such as synaptogenesis, neuroinflammation and dendritic plasticity may be involved in depressive disorders and these factors still remain unclear in the study of neuropathology of depression [47]. Furthermore, as the majority of discoveries were found in preclinical situations, studies with human subjects or tissues will greatly facilitate the understanding of the issue [10]. Far more efforts will be required to elucidate the roles of neurogenesis in depressive disorders, and such insight will encourage future development of novel treatments.

\section{Neurogenesis and anxiety disorders}

Information regarding the involvement of neurogenesis in anxiety disorders was mainly generated from preclinical studies using animal models. The study of anxiety-like 
behaviors in animals was originally related to animal models of depressive disorder. Application of stress is a direct cause of anxiety and it is widely considered as a risk factor of major depression and thus it is commonly used to establish animal models of depression. Despite the effort to study the relationship between neurogenesis and anxiety, several issues remain to be solved before a hypothesis regarding anxiety disorders and neurogenesis could be formulated. There is, surprisingly, still lack of clinical studies which examine neurogenesis in patients with anxiety disorders (including diagnosed generalized anxiety disorders, phobia and panic disorders) and this makes the significance of neurogenesis in these patients elusive. Another issue is that stressed animal models were usually used for the study of both anxiety and depression-like behaviors [48], which indicates that the two types of behaviors are not usually clearly defined in experimental designs. Since depressionlike behavior may interfere with anxiety-like behaviors and vice versa, the roles of neurogenesis in animal studies using anxiety models may be masked by depression-like behaviors.

The association between neurogenesis and anxiety was suggested after Malberg et al. [9] discovered the proneurogenic effect of antidepressants as this class of medication was also used to treat anxiety disorders. As mentioned above, animal models used for the studies usually employ various types of stress to elicit anxiety responses, such as novelty-suppressed feeding (NSF) paradigm, unpredictable chronic mild stress (CMS) paradigm and social defeat test $[30,49,50]$. These tests induced stressful situations from behavioral or environmental measures and the variability of experimental conditions among different laboratories produced difficulties in the replication of results. In order to avoid this issue, several groups used exogenously applied corticosterone to induce anxiety-like behavior [18,51-53], since corticosterone is an adrenal hormone which is produced when the individual is under stressful situations such as restraint stress and exposure to predator. The dysfunction of the HPA-axis and hypercortisolemia found in depressive patients also support the application of corticosterone to simulate depressive/anxious state in animals [54,55]. In short, various animal models were used to study anxiety-like behavior and this may be one of the causes of inconsistent findings across different studies.

A hallmark study conducted by Santarelli et al. [30] indicates the requirement of neurogenesis in therapeutic effect of antidepressants. In non-stressed mice, the latency to feed in NSF paradigm (which indicates the anxiety-like behavior) was decreased by chronic treatment of fluoxetine, which is associated with increased neurogenesis. When the dentate gyrus neurogenesis was ablated by $\mathrm{X}$-irradiation, the anxiolytic effect of fluoxetine was abolished. Inspired by this finding, later studies examined whether the anxiolytic effect of antidepressants is neurogenesis dependent or independent. Contradictory results were found among different reports. HU210, a synthetic cannabinoid, exerts anxiolytic and anti- depressant effects on animals while the effect was abolished if hippocampal neurogenesis was blocked [33]. In contrast, the anxiolytic effect of environmental enrichment and voluntary running was not affected by the blocking of neurogenesis [56]. Furthermore, the anxiolytic effect of a melaninconcentrating hormone receptor antagonist was found to be neurogenesis-independent while a recent study shows that pregabalin, an $\alpha 2 \delta$ ligand used for the treatment of neuropathic pain and generalized anxiety disorders, prevented stress-induced depression-like behaviors and promotes the generation of neural precursor cells [57]. The discrepancy in the findings depends on the choice of behavior tests and method to abolish neurogenesis [51], in which forced swimming test and open field test are neurogenesis independent while NSF and coat state are neurogenesis-dependent. As great differences between choice of stress model, method of blocking neurogenesis, behavioral testing and type of drugs used for treatment are commonplace, significance of neurogenesis in anxiety and treatment effect requires further investigations.

Unlike other anxiety disorders, post-trauma stress disorder (PTSD) was shown to associate with hippocampal neurogenesis from clinical reports, although the number of reports is still limited. Patients suffered from PTSD showed hippocampal atrophy and deficits in declarative verbal memory, which is related to hippocampus, while chronic treatment with paroxetine (a SSRI which increases neurogenesis $[58,59]$ ) could improve verbal declarative memory and reversed the hippocampal atrophy [60]. The effect of treatment for PTSD with another epileptic drug was replicated [61] in another study. From an animal study simulating PTSD situation, inescapable shocks (IS) were applied on rats and PTSD-like (hypervigilant/hyperarousal) responses were elicited [62]. Hippocampal neurogenesis was decreased and it was correlated with the hyperactive behavioral parameters. Chronic SSRI treatment was able to rescue the decreased neurogenesis and decrease the hyperactive behavior simultaneously. Mechanistically, the disturbance of BDNF signaling in PTSD patients suggested a possible connection between neurogenesis and PTSD condition [63]. Although the data about the relationship between PTSD and neurogenesis seem to be promising, the hypothesis needs to be further confirmed by more in-depth pre-clinical and clinical studies.

\section{Neurogenesis and schizophrenia}

The association between schizophrenia and neurogenesis is supported by both clinical and pre-clinical reports. According to a post-mortem study conducted by Reif et al. [26], the density of Ki-67 (a nuclear antigen expressed by proliferative cells) in the dentate gyrus of schizophrenia patients was $60 \%$ lower than that of control subjects. Surprisingly samples from bipolar disorder and major depression patients did not 
show a significant lower cell density than the controls. This is the first clinical study which directly showed the alteration of neurogenesis in schizophrenic patients. Although the result was clear and direct, there are several points that should be considered when interpreting the results. First, since Ki-67 expression is not restricted to proliferative cells of neuronal lineage, the Ki-67 quantification could not be directly interpreted as quantifying neuronal proliferation [64]. Glial cells such as astrocytes in the dentate gyrus may be quantified too. Second, the result only represented the initial stage of neurogenesis. As the majority of newly proliferative neural precursor cells die before maturation, only a certain proportion of new neurons survives and becomes functional. Thus the later stages of neurogenesis (including survival, differentiation and integration into the neural circuit) should be investigated. Third, the result in the study was expressed as cell density. Since emotional disorder and schizophrenia were characterized by a reduction in hippocampal volume [64-67], the expression of result in terms of density could not show the absolute number of proliferative cells in samples being investigated.

Being similar to depressive disorders, further evidence to support the link between neurogenesis and schizophrenia is provided by pre-clinical studies of pharmacological treatments related to the disorder. Phencyclidine, a hallucinogenic drug which induces symptoms similar to schizophrenia, reduces neurogenesis in animals [68]. Antipsychotics, in general, increase neurogenesis in animal models while the effect may be type and species-specific. Prototypic antipsychotic haloperidol promotes neurogenesis in the dentate gyrus of gerbils [69], while there is no effect in rats [70]. Atypical antipsychotics olanzapine and clozapine were also shown to increase neural stem cell proliferation in different regions including the dorsal striatum, hippocampus and prefrontal cortex [70-72]. Although other studies showed contradictory results $[73,74]$, the discrepancies may be due to animal species used, dosage of drug chosen, treatment regime and route of administration. Thus the effect of antipsychotics on neurogenesis could not be ignored.

An interesting finding that distinguishes schizophrenia from other psychiatric illness, in the view point of neurogenesis, is the existence of single genes susceptible to schizophrenia which are closely related to neurogenesis. One of the candidate genes under intensive investigation is DISC1 (disrupted in schizophrenia 1) [75]. This gene was discovered in a Scottish family with a large spectrum of psychiatric disorders, and it is related to different traits including working memory, cognitive performance and gray matter volume. Genetic analysis and abnormal expression level in schizophrenic patients [73,74] support the strong linkage between DISC1 and schizophrenia. Interestingly, DISC1 is involved in several neuronal functions such as neuronal migration, neurite outgrowth and synaptic plasticity, which are essential processes in neurogenesis and neurodevelopment $[11,76]$. In transgenic mice with Disc1 (mouse orthology of DISC1) mutation, working memory was decreased [77]. In the adult brain, down-regulation of Disc1 led to aberrant cell morphology, accelerated growth of axons and dendrites, ectopic location of new neurons [78], shorter dendrites and decreased surface area [79], and changes in the plasticity of mossy fiber/CA3 circuit [80]. Functionally, the immature neurons with low Disc1 expression showed exaggerated excitability and synaptic formation. These findings illustrate the significance of Disc1 in the process of neurogenesis, especially during the stage of integration of immature neurons into the existing circuitry. Since Disc1 has different binding partners to exert its function, it is possible that misregulation of the other binding proteins also contribute to the abnormal neurogenesis process, and contribute to the disease process [81].

Recent studies disclosed the mechanism of neurogenesis regulation by DISC1. NMDA receptor, a receptor of glutamate, is essential for proper newborn neuron migration and integration [82]. Memantine (a NMDA receptor antagonist) was shown to decrease the expression level of DISC1 and cause abnormal positioning of new neurons in the dentate gyrus, while overexpression of DISC1 could prevent the abnormalities. GABA-induced depolarization of neural progenitor cells was recently found to be the pathway involved in DISC1 action [83], with convergence onto AKT (protein kinase B) pathway. DISC1 regulates depolarization induced by GABA signaling, which in turn controls the dendritic growth. When the time period of depolarization to hyperpolarization was accelerated, DISC1 loses the control of dendritic growth. In contrast, extension of this period allows DISC1 to regain regulation of dendritic growth. If DISC1 is suppressed, one of its binding partners KIAA1212 enhances activation of AKT, which leads to improper positioning and dendritic growth of newborn neurons. The defects could be prevented by the inhibition of an AKT downstream effector, mammalian target of rapamycin (mTOR) [84]. Taken together, the regulation of neurogenesis by DISC1 involves neurotransmitter such as GABA and glutamate, molecular switch such as AKT and mTOR and DISC1 binding partners such as KIAA1212. The underlying mechanisms are being revealed, and further findings will definitely help to understand the regulation of neurogenesis, dendritic aberration in schizophrenia condition.

Neuronal PAS domain-containing protein 3 (NPAS3) is another worth noting gene with susceptibility of schizophrenia [85]. NPAS3 is a basic helix-loop-helix transcription factor which was discovered in a family that suffered from schizophrenia [85]. Transgenic mice with NPAS3 mutation showed a behavioral phenotype with reduced memory, impaired social recognition and impaired sensorimotor gating [86], which are also characteristics of schizophrenic patients. These animals simultaneously had a reduced neurogenesis in the dentate gyrus [87]. Aminopropyl carbazole, which promotes neurogenesis by preventing apoptosis in the neurogenesis process, was able to increase neurogenesis and 
reversed the malformation of dentate gyrus in NPAS3 knock-out animals [86]. This suggests the proneurogenic property of NPAS3 lies on prevention of cell death but not proliferation. Further assessment of the association between NPAS3, neurogenesis and schizophrenia may provide another possible target of future treatment.

Another schizophrenia susceptibility gene is neuregulin 1 (NRG1) [88]. This pleiotropic growth factor is important during neurodevelopment and functioning. If signaling pathway of NRG1 is disturbed, $N$-meth7yl- $D$-aspartic acid (NMDA) receptor dysfunction may occur. This is hypothesized to be the central mechanism underlying schizophrenia. The proneurogenic roles of NRG1 and neuregulin 2 (NRG2) were reported in the subventricular zone, while their roles in hippocampal neurogenesis remain to be determined [89]. Although not yet clear, NRG1 and NRG2 may also be involved in neuron production in the dentate gyrus and the positive signs and symptoms of schizophrenia.

As prenatal infection is a well accepted risk factor of schizophrenia, several research groups utilized an animal model of prenatal infection/maternal immune activation to study the involvement of neurogenesis in schizophrenia. Lipopolysaccharide (LPS) and polyriboinosinic-polyribocytidilic acid (polyI:C) were used to simulate bacterial and viral effects in pregnancy, and behavioral, chemical and cognitive changes are found in the animals, including disruption of prepulse inhibition, decrease in attention and working memory and responses to antipsychotic drugs [90,91]. The neurogenesis of the polyI:C treated offspring in adulthood was decreased [92], while co-treatment with risperidone could prevent such decrease [93]. These collectively suggests that persistent changes in adult neurogenesis and cognitive functions could be induced by prenatal immune challenge at early developmental stages, and the maternal immune activation models may provide interesting insight for the pathogenesis of schizophrenia.

Although it is unlikely that schizophrenia is caused by single genetic disruption or mutation, the studying of susceptibility genes will illustrate different facets of the schizophrenia pathophysiology. Given that neurogenesis is closely associated with the candidate genes and disruption of neurogenesis is found in clinical and pre-clinical reports, hypothesis related to neurogenesis may provide a novel insight on the etiology and disease progress of schizophrenia. It is speculated that the altered neurogenesis may contribute to disrupted cognition, memory or social interaction in schizophrenia, while these require further evidence to support [7].

\section{Amygdala and cortex: Non-traditional neu- rogenic regions and relationship with psychiatric conditions}

The dentate gyrus of the hippocampus and the subventricular zone (SVZ) are the two well-accepted traditional regions which have the ability to produce new neurons in the adult brain. Several regions, such as the amygdala and the neocortex, have been claimed to be the non-traditional neurogenic brain regions owing to the controversial results of their ability to produce new neurons in the adult brain [94-97]. Though the possibility of adult neurogenesis in these regions is still under debate, studies on the cellular changes within the non-traditional neurogenic brain regions in relation to psychiatric illness should also be focused in addition to the vast majority of studies focusing on the traditional neurogenic sites. Just because the non-traditional neurogenic regions, such as the amygdala and neocortex, also play an important role in mediating various psychological behaviors: social behaviors, reproductive behaviors, learning and memory [95]. The cellular changes within these regions may thus be highly related to the pathophysiology of the psychiatric disorder.

Amygdala is one of the important structures within the limbic system and composed of different nuclei including basolateral, medial and central nuclei. These different parts of the nuclei responsible for different responses upon stimulus from the environment and overall contribute to the social processes and emotions of individuals [98-100]. Recently, a study reported that upon chronic social isolation of the female prairie voles from the main group could cause reduction in cell proliferation and alter neuronal differentiation within the amygdala and similar results were obtained within the dentate gyrus of the hippocampus. These results support the fact that neurogenesis may be possible in the non-neurogenic site. Moreover, the reduction of the proliferating cells in the amygdala seems to be associated with the behavioral changes. For example, the isolated female voles were found to have a higher anxiety level when compared to the non-isolated group such that they have fewer entries to the center zone of the open-field test and a lower level of new cells was found concurrently within the amygdala [96]. This report has provided evidence that new neurons can be produced within the amygdala which is not one of the two neurogenic sites. In addition, the functional significance of the cells found in the amygdala might link to regulating the anxiety upon the environment stimulus. Other than the decrease of neurogenesis in the amygdala can cause increase in the anxiety level, autism has also been hypothesized to be related to neurogenesis in the amygdala [99]. Autism is a disorder in which the patients often show an unwillingness to have any social interactions $[99,101]$. Based on the observation on the morphological changes of the amygdala, it was found that there was a significantly decreased volume of the patients with autism [102]. The volume change of the amygdala may be due to decrease in neurogenesis or decreased in pruning of neurons $[99,103]$. Though some other controversial results have also shown, these results have given the possible relationship between the morphological changes of the amygdala and the pathophysiologic development of autism. Mercadante et al. [99] 
Table 1 Summary of findings supporting the involvement of neurogenesis in psychiatric conditions

\begin{tabular}{|c|c|c|c|}
\hline & Animal study & Clinical study & Molecule/pathways involved \\
\hline Major depression & $\begin{array}{l}\text {-Stress (risk factor of depression): } \\
\text { Suppress neurogenesis [30] } \\
\text {-Antidepressants increases neuro- } \\
\text { genesis }[9,23] \\
\text {-Intact neurogenesis required for } \\
\text { treatment effect of antidepres- } \\
\text { sants and recovery of HPA axis } \\
{[30,34]}\end{array}$ & $\begin{array}{l}\text {-Post-mortem brain study: Antide- } \\
\text { pressant treatment increase neuro- } \\
\text { genesis and angiogenesis [27] } \\
\text {-Decrease in cortical gliogenesis } \\
\text { associated with major depression } \\
{[110,111]}\end{array}$ & $\begin{array}{l}\text { Growth factors: } \\
\text { 1) Brain-derived neurotrophic factor (BDNF) [31] } \\
\text { 2) Vascular endothelial growth factor (VEGF) [41] } \\
\text { 3) Insulin-like growth factor } 1 \text { (IGF1) [41] } \\
\text { Neurotransmitters: } \\
\text { 1) GABA [36] } \\
\text { 2) Serotonin [37] } \\
\text { 3) Glutamate/NMDA receptor [38] } \\
\text { 4) Dopamine and acetylcholine [39] } \\
\text { Immunoregulatory molecules: } \\
\text { 1) Macrophage migration inhibitory factor (MIF) [43] } \\
\text { 2) Nuclear factor kappa-light-chain-enhancer of ac- } \\
\text { tivated B cells (NF- } \mathrm{kB} \text { ) [42] }\end{array}$ \\
\hline Anxiety Disorders & $\begin{array}{l}\text {-Animal models of anxiety disor- } \\
\text { ders: Show decreased neurogen- } \\
\text { esis [30,49] } \\
\text {-Anxiolytic effect of antidepres- } \\
\text { sant depends on intact neurogen- } \\
\text { esis [30] } \\
\text {-Pregabalin (Treatment for gener- } \\
\text { alized anxiety disorder) promote } \\
\text { neurogenesis [57] } \\
\text {-Animal model of PTSD: Show } \\
\text { decreased neurogenesis [57] }\end{array}$ & $\begin{array}{l}\text {-PTSD patients: show hippocampal } \\
\text { atrophy and deficits in hippocam- } \\
\text { pus-dependent verbal memory [63] }\end{array}$ & Brain-derived neurotrophic factor (BDNF) [63] \\
\hline Schizophrenia & $\begin{array}{l}\text {-Phencyclidine (Hallucinogenic } \\
\text { drug) suppress neurogenesis [68] } \\
\text {-Antipsychotics increases neuro- } \\
\text { genesis [68] }\end{array}$ & $\begin{array}{l}\text {-Decrease in number of Ki-67 +ve } \\
\text { cells in schizophrenia patients [26] }\end{array}$ & $\begin{array}{l}\text { Intracellular molecules: } \\
\text { 1) Disrupted in Schizophrenia } 1 \text { (DISC1) [75] } \\
\text { 2) Neuronal PAS domain protein } 3 \text { (NPAS3) [85] } \\
\text { 3) Neuregulin 1 (NRG1) [88] } \\
\text { Neurotransmitters: } \\
\text { 1) Glutamate [82] } \\
\text { 2) GABA [83] } \\
\text { Pathways: } \\
\text { 1) AKT/mTOR pathway [84] } \\
\text { Immunoregulation [92] }\end{array}$ \\
\hline
\end{tabular}

have further suggested that the new immature-neurons newly produced in the amygdala may be helpful in increasing the ability to learn from new experiences via modulation of the gamma-aminobutyric acid (GABA) as patients with autism were found to have alteration on the GABAergic system. However, further investigations are needed as this is a new etiologic hypothesis of autism.

Neocortex is another non-traditional neurogenic region which has been highly debated about the existence of adult neurogenesis within this region. Gould et al. [104] was the first group to report that new neurons could be found in the adult primate using macaque monkeys. In addition, they have also suggested that the new neurons were from the subventricular zone and these migrating cells bypass the corpus callosum to reach the cerebral cortex [104]. There were some other studies supporting the above findings such that the new cells found within the neocortex of primates were originated from the subventricular zone, though under pathological conditions only, such as ischemia [105,106]. A recent study has shown that new cells could be found in layer I of the mice's neocortex, though under ischemic con- ditions. Further tracing the proliferating cells found in layer I of the neocortex with the membrane-targeted green fluorescence proteins, these cells were shown to migrate to the other cerebral layers and further differentiate into GABAergic neurons [107]. This study has suggested that neurogenesis may occur locally within layer I of the neocortex, but other origin of these new cells should not be excluded. However, the concept of neurogenesis within the adult neocortex has been challenged by many other groups. For example, Kornack et al. [108] have applied the injection of bromodeoxyuridine (BrdU) to trace the proliferating cells in macaque monkeys and found that none of the BrdU positive cells has double-labeled with the neuronal markers. Although many studies have shown whether new cells could be found within the neocortex, there were only few studies focused on the functional significance of the new cells found in the neocortex and the possible relations with the psychiatric disorders. Compared to the cortical neurogenesis, gliogenesis seems to have more studies reporting about the changes of the glial cells within the neocortex and the possible role in relation with psychiatric disorders. For example, 
depressive-like behaviors were found in adult rats using a pharmacologic glial ablation technique in the prefrontal cortex which suggested the possible role of the glial cells found in the prefrontal cortex in relation to depression [109]. Another example has shown that chronic stress could decrease cell proliferation in the medial prefrontal cortex which cause abnormal decrease in glial cells number [110]. According to some of the human studies, the decrease in the number of glial cells in the prefrontal cortex can trigger the development of various mood disorders [110,111]. Also, there was a study that found the reduction in glial density in the anterior cingulated cortex may be related to the major depressive disorder [111]. Glial cells have been previously suggested to retain the ability to renew in adult brains [110] and they have been shown to have important roles such as: regulating the process of synaptogenesis, adjusting the cerebral flow and also lying very close to the neurons and the blood vessels for mediating the process of neurogenesis and angiogenesis [111]. Although neurogenesis is known to have an important role in relation to the psychiatric disorders, the study on the gliogenesis within the adult brain should not be excluded particularly within the non-traditional neurogenic sites.

\section{Conclusion}

Altered adult neurogenesis is widely observed in various animal models of psychiatric illnesses (Summarized in Table 1). Ablation of neurogenesis reveals that neurogenesis could not explain the entire pathophysiology of different disorders, and the consequence of altered neurogenesis in the animal models requires further examination. While suppression of neurogenesis is not a sufficient criterion for the development of depression, its association with schizophrenia and certain anxiety disorders begins to emerge from both clinical and pre-clinical studies. To further elucidate the roles of neurogenesis in psychiatric illnesses, clinical studies which assess the symptoms and functions related to traditional neurogenic zone (hippocampus and subventricular zone) and non-traditional neurogenic ones (e.g. amygdala and neocortex) are necessary, and development of standardized animal model/paradigm will be beneficial for solving the puzzle.

This work was supported by funding from the Jessie Ho Professorship in Neuroscience (The University of Hong Kong for Educational Development and Research Limited) and the Fundamental Research Funds for the Central Universities (21609101).

1 Kessler R C, Chiu W T, Demler O, et al. Prevalence, severity, and comorbidity of 12-month DSM-IV disorders in the National Comorbidity Survey Replication. Arch Gen Psychiatry, 2005, 62: 617-627

2 DeCarolis N A, Eisch A J. Hippocampal neurogenesis as a target for the treatment of mental illness: A critical evaluation. Neuropharmacology, 2010, 58: 884-893
3 Eisch A J. Adult neurogenesis: Implications for psychiatry. Prog Brain Res, 2002, 138: 315-342

4 Bast T. Toward an integrative perspective on hippocampal function: From the rapid encoding of experience to adaptive behavior. Rev Neurosci, 2007, 18: 253-281

5 Eichenbaum H, Fortin N J. The neurobiology of memory based predictions. Philos Trans R Soc Lond B Biol Sci, 2009, 364: 1183-1191

6 Fuchs E, Flugge G. Stress, glucocorticoids and structural plasticity of the hippocampus. Neurosci Biobehav Rev, 1998, 23: 295-300

7 Kempermann G, Krebs J, Fabel K. The contribution of failing adult hippocampal neurogenesis to psychiatric disorders. Curr Opin Psychiatry, 2008, 21: 290-295

8 Sahay A, Hen R. Adult hippocampal neurogenesis in depression. Nat Neurosci, 2007, 10: 1110-1115

9 Malberg J E, Eisch A J, Nestler E J, et al. Chronic antidepressant treatment increases neurogenesis in adult rat hippocampus. J Neurosci, 2000, 20: 9104-9110

10 Samuels B A, Hen R. Neurogenesis and affective disorders. Eur J Neurosci, 2011, 33: 1152-1159

11 Ming G L, Song H. Adult neurogenesis in the mammalian brain: Significant answers and significant questions. Neuron, 2011, 70: 687702

12 Altman J, Das G D. Autoradiographic and histological evidence of postnatal hippocampal neurogenesis in rats. J Comp Neurol, 1965, 124: 319-335

13 Paton J A, O'Loughlin B E, Nottebohm F. Cells born in adult canary forebrain are local interneurons. J Neurosci, 1985, 5: 3088-3093

14 Pencea V, Bingaman K D, Wiegand S J, et al. Infusion of brain-derived neurotrophic factor into the lateral ventricle of the adult rat leads to new neurons in the parenchyma of the striatum, septum, thalamus, and hypothalamus. J Neurosci, 2001, 21: 6706-6717

15 Kempermann G, Wiskott L, Gage F H. Functional significance of adult neurogenesis. Curr Opin Neurobiol, 2004, 14: 186-191

16 Shors T J, Miesegaes G, Beylin A, et al. Neurogenesis in the adult is involved in the formation of trace memories. Nature, 2001, 410: 372-376

17 Mak G K, Enwere E K, Gregg C, et al. Male pheromone-stimulated neurogenesis in the adult female brain: Possible role in mating behavior. Nat Neurosci, 2007, 10: 1003-1011

18 Lau B W, Yau S Y, Lee T M, et al. Effect of corticosterone and paroxetine on masculine mating behavior: Possible involvement of neurogenesis. J Sex Med, 2011, 8: 1390-1403

19 Gould E, McEwen B S, Tanapat P, et al. Neurogenesis in the dentate gyrus of the adult tree shrew is regulated by psychosocial stress and NMDA receptor activation. J Neurosci, 1997, 17: 2492-2498

20 Dagyte G, Crescente I, Postema F, et al. Agomelatine reverses the decrease in hippocampal cell survival induced by chronic mild stress. Behav Brain Res, 2011, 218: 121-128

21 Racagni G, Riva M A, Molteni R, et al. Mode of action of agomelatine: Synergy between melatonergic and 5-HT2C receptors. World J Biol Psychiatry, 2011, 12: 574-587

22 Kohl Z, Winner B, Ubhi K, et al. Fluoxetine rescues impaired hippocampal neurogenesis in a transgenic A53T synuclein mouse model. Eur J Neurosci, 2012, 35: 10-19

23 Banasr M, Duman R S. Regulation of neurogenesis and gliogenesis by stress and antidepressant treatment. CNS Neurol Disord Drug Targets, 2007, 6: 311-320

24 Meltzer L A, Yabaluri R, Deisseroth K. A role for circuit homeostasis in adult neurogenesis. Trends Neurosci, 2005, 28: 653-660

25 Drew M R, Hen R. Adult hippocampal neurogenesis as target for the treatment of depression. CNS Neurol Disord Drug Targets, 2007, 6: 205-218

26 Reif A, Fritzen S, Finger M, et al. Neural stem cell proliferation is decreased in schizophrenia, but not in depression. Mol Psychiatry, 2006, 11: 514-522

27 Boldrini M, Hen R, Underwood M D, et al. Hippocampal angiogenesis and progenitor cell proliferation are increased with antidepressant use in major depression. Biol Psychiatry, 2012, 72: 562-571

28 Airan R D, Meltzer L A, Roy M, et al. High-speed imaging reveals neurophysiological links to behavior in an animal model of depres- 
sion. Science, 2007, 317: 819-823

29 David D J, Samuels B A, Rainer Q, et al. Neurogenesis-dependent and -independent effects of fluoxetine in an animal model of anxiety/depression. Neuron, 2009, 62: 479-493

30 Santarelli L, Saxe M, Gross C, et al. Requirement of hippocampal neurogenesis for the behavioral effects of antidepressants. Science, 2003, 301: 805-809

31 Li Y, Luikart B W, Birnbaum S, et al. TrkB regulates hippocampal neurogenesis and governs sensitivity to antidepressive treatment. Neuron, 2008, 59: 399-412

32 Taliaz D, Stall N, Dar D E, et al. Knockdown of brain-derived neurotrophic factor in specific brain sites precipitates behaviors associated with depression and reduces neurogenesis. Mol Psychiatry, 2010, 15: 80-92

33 Jiang W, Zhang Y, Xiao L, et al. Cannabinoids promote embryonic and adult hippocampus neurogenesis and produce anxiolytic- and antidepressant-like effects. J Clin Invest, 2005, 115: 3104-3116

34 Snyder J S, Soumier A, Brewer M, et al. Adult hippocampal neurogenesis buffers stress responses and depressive behaviour. Nature, 2011, 476: 458-461

35 Surget A, Tanti A, Leonardo E D, et al. Antidepressants recruit new neurons to improve stress response regulation. Mol Psychiatry, 2011, 16: $1177-1188$

36 Tozuka Y, Fukuda S, Namba T, et al. GABAergic excitation promotes neuronal differentiation in adult hippocampal progenitor cells. Neuron, 2005, 47: 803-815

37 Diaz S L, Doly S, Narboux-Neme N, et al. 5-HT(2B) receptors are required for serotonin-selective antidepressant actions. Mol Psychiatry, 2012, 17: 154-163

38 Nacher J, McEwen B S. The role of $N$-methyl- $D$-asparate receptors in neurogenesis. Hippocampus, 2006, 16: 267-270

39 Veena J, Rao B S, Srikumar B N. Regulation of adult neurogenesis in the hippocampus by stress, acetylcholine and dopamine. J Nat Sci Biol Med, 2011, 2: 26-37

40 Warner-Schmidt J L, Duman R S. Hippocampal neurogenesis: Opposing effects of stress and antidepressant treatment. Hippocampus, 2006, 16: 239-249

41 Warner-Schmidt J L, Duman R S. VEGF is an essential mediator of the neurogenic and behavioral actions of antidepressants. Proc Natl Acad Sci USA, 2007, 104: 4647-4652

42 Wang Y, Cui X L, Liu Y F, et al. LPS inhibits the effects of fluoxetine on depression-like behavior and hippocampal neurogenesis in rats. Prog Neuropsychopharmacol Biol Psychiatry, 2011, 35: 1831-1835

43 Conboy L, Varea E, Castro J E, et al. Macrophage migration inhibitory factor is critically involved in basal and fluoxetine-stimulated adult hippocampal cell proliferation and in anxiety, depression, and memory-related behaviors. Mol Psychiatry, 2011, 16: 533-547

44 Zunszain P A, Anacker C, Cattaneo A, et al. Interleukin-1beta: A new regulator of the kynurenine pathway affecting human hippocampal neurogenesis. Neuropsychopharmacology, 2012, 37: 939-949

45 Pickard B. Progress in defining the biological causes of schizophrenia. Expert Rev Mol Med, 2011, 13: e25

46 Tang S W, Helmeste D, Leonard B. Is neurogenesis relevant in depression and in the mechanism of antidepressant drug action? A critical review. World J Biol Psychiatry, 2011, 13: 402-412

47 Chen F, Madsen T M, Wegener G, et al. Imipramine treatment increases the number of hippocampal synapses and neurons in a genetic animal model of depression. Hippocampus, 2010, 20: 1376-1384

48 Petrik D, Lagace D C, Eisch A J. The neurogenesis hypothesis of affective and anxiety disorders: Are we mistaking the scaffolding for the building? Neuropharmacology, 2012, 62: 21-34

49 Berton O, McClung C A, Dileone R J, et al. Essential role of BDNF in the mesolimbic dopamine pathway in social defeat stress. Science, 2006, 311: 864-868

50 Dulawa S C, Holick K A, Gundersen B, et al. Effects of chronic fluoxetine in animal models of anxiety and depression. Neuropsychopharmacology, 2004, 29: 1321-1330

51 David D J, Wang J, Samuels B A, et al. Implications of the functional integration of adult-born hippocampal neurons in anxiety-depression disorders. Neuroscientist, 2010, 16: 578-591

52 Ardayfio P, Kim K S. Anxiogenic-like effect of chronic corticosterone in the light-dark emergence task in mice. Behav Neurosci, 2006, 120: 249-256

53 Murray F, Smith D W, Hutson P H. Chronic low dose corticosterone exposure decreased hippocampal cell proliferation, volume and induced anxiety and depression like behaviours in mice. Eur J Pharmacol, 2008, 583: 115-127

54 Holsboer F. How can we realize the promise of personalized antidepressant medicines? Nat Rev Neurosci, 2008, 9: 638-646

55 Gourley S L, Wu F J, Kiraly D D, et al. Regionally specific regulation of ERK MAP kinase in a model of antidepressant-sensitive chronic depression. Biol Psychiatry, 2008, 63: 353-359

56 Meshi D, Drew M R, Saxe M, et al. Hippocampal neurogenesis is not required for behavioral effects of environmental enrichment. Nat Neurosci, 2006, 9: 729-731

57 Valente M M, Bortolotto V, Cuccurazzu B, et al. Alpha2delta ligands act as positive modulators of adult hippocampal neurogenesis and prevent depression-like behavior induced by chronic restraint stress. Mol Pharmacol, 2012, 82: 271-280

58 Lau W M, Qiu G, Helmeste D M, et al. Corticosteroid decreases subventricular zone cell proliferation, which could be reversed by paroxetine. Restor Neurol Neurosci, 2007, 25: 17-23

59 Qiu G, Helmeste D M, Samaranayake A N, et al. Modulation of the suppressive effect of corticosterone on adult rat hippocampal cell proliferation by paroxetine. Neurosci Bull, 2007, 23: 131-136

60 Vermetten E, Vythilingam M, Southwick S M, et al. Long-term treatment with paroxetine increases verbal declarative memory and hippocampal volume in posttraumatic stress disorder. Biol Psychiatry, 2003, 54: 693-702

61 Bremner J D. The relationship between cognitive and brain changes in posttraumatic stress disorder. Ann N Y Acad Sci, 2006, 1071: $80-86$

62 Kikuchi A, Shimizu K, Nibuya M, et al. Relationship between posttraumatic stress disorder-like behavior and reduction of hippocampal 5-bromo-2'-deoxyuridine-positive cells after inescapable shock in rats. Psychiatry Clin Neurosci, 2008, 62: 713-720

63 Kaplan G B, Vasterling J J, Vedak P C. Brain-derived neurotrophic factor in traumatic brain injury, post-traumatic stress disorder, and their comorbid conditions: Role in pathogenesis and treatment. Behav Pharmacol, 2010, 21: 427-437

64 Eisch A J, Mandyam C D. Adult neurogenesis: Can analysis of cell cycle proteins move us "Beyond BrdU"? Curr Pharm Biotechnol, 2007, 8: 147-165

65 Levitt J J, Bobrow L, Lucia D, et al. A selective review of volumetric and morphometric imaging in schizophrenia. Curr Top Behav Neurosci, 2010, 4: 243-281

66 Videbech P, Ravnkilde B. Hippocampal volume and depression: A meta-analysis of MRI studies. Am J Psychiatry, 2004, 161: 19571966

67 Davidson C A, Kuroki N, Alvarado J L, et al. An MRI study of septi pellucidi in relation to hippocampus volume and fornix integrity in schizophrenia. Schizophr Res, 2012, 134: 165-170

68 Liu J, Suzuki T, Seki T, et al. Effects of repeated phencyclidine administration on adult hippocampal neurogenesis in the rat. Synapse, 2006, 60: 56-68

69 Dawirs R R, Hildebrandt K, Teuchert-Noodt G. Adult treatment with haloperidol increases dentate granule cell proliferation in the gerbil hippocampus. J Neural Transm, 1998, 105: 317-327

70 Halim N D, Weickert C S, McClintock B W, et al. Effects of chronic haloperidol and clozapine treatment on neurogenesis in the adult rat hippocampus. Neuropsychopharmacology, 2004, 29: 1063-1069

71 Kodama M, Fujioka T, Duman R S. Chronic olanzapine or fluoxetine administration increases cell proliferation in hippocampus and prefrontal cortex of adult rat. Biol Psychiatry, 2004, 56: 570-580

72 Wang H D, Dunnavant F D, Jarman T, et al. Effects of antipsychotic drugs on neurogenesis in the forebrain of the adult rat. Neuropsychopharmacology, 2004, 29: 1230-1238

73 Schmitt A, Weber S, Jatzko A, et al. Hippocampal volume and cell 
proliferation after acute and chronic clozapine or haloperidol treatment. J Neural Transm, 2004, 111: 91-100

74 Wakade C G, Mahadik S P, Waller J L, et al. Atypical neuroleptics stimulate neurogenesis in adult rat brain. J Neurosci Res, 2002, 69: $72-79$

75 Hennah W, Thomson P, Peltonen L, et al. Beyond schizophrenia: The role of DISC1 in major mental illness. Schizophr Bull, 2006, 32: 409-416

76 Enomoto A, Asai N, Namba T, et al. Roles of disrupted-in-schizophrenia 1-interacting protein girdin in postnatal development of the dentate gyrus. Neuron, 2009, 63: 774-787

77 Koike H, Arguello P A, Kvajo M, et al. Disc1 is mutated in the $129 \mathrm{~S} 6 / \mathrm{SvEv}$ strain and modulates working memory in mice. Proc Natl Acad Sci USA, 2006, 103: 3693-3697

78 Duan X, Chang J H, Ge S, et al. Disrupted-In-Schizophrenia 1 regulates integration of newly generated neurons in the adult brain. Cell, 2007, 130: 1146-1158

79 Lee F H, Fadel M P, Preston-Maher K, et al. Disc1 point mutations in mice affect development of the cerebral cortex. J Neurosci, 2011, 31: 3197-3206

80 Kvajo M, McKellar H, Drew L J, et al. Altered axonal targeting and short-term plasticity in the hippocampus of Disc1 mutant mice. Proc Natl Acad Sci USA, 2011, 108: E1349-1358

81 Ikeda M, Hikita T, Taya S, et al. Identification of $Y W H A E$, a gene encoding 14-3-3epsilon, as a possible susceptibility gene for schizophrenia. Hum Mol Genet, 2008, 17: 3212-3222

82 Namba T, Ming G L, Song H, et al. NMDA receptor regulates migration of newly generated neurons in the adult hippocampus via Disrupted-In-Schizophrenia 1 (DISC1). J Neurochem, 2011, 118: 34-44

83 Kim J Y, Liu C Y, Zhang F, et al. Interplay between DISC1 and GABA signaling regulates neurogenesis in mice and risk for schizophrenia. Cell, 2012, 148: 1051-1064

84 Kim J Y, Duan X, Liu C Y, et al. DISC1 regulates new neuron development in the adult brain via modulation of AKT-mTOR signaling through KIAA1212. Neuron, 2009, 63: 761-773

85 Kamnasaran D, Muir W J, Ferguson-Smith M A, et al. Disruption of the neuronal PAS3 gene in a family affected with schizophrenia. J Med Genet, 2003, 40: 325-332

86 Erbel-Sieler C, Dudley C, Zhou Y, et al. Behavioral and regulatory abnormalities in mice deficient in the NPAS1 and NPAS3 transcription factors. Proc Natl Acad Sci USA, 2004, 101: 13648-13653

87 Pieper A A, Xie S, Capota E, et al. Discovery of a proneurogenic, neuroprotective chemical. Cell, 2010, 142: 39-51

88 Harrison P J, Law A J. Neuregulin 1 and schizophrenia: Genetics, gene expression, and neurobiology. Biol Psychiatry, 2006, 60: 132140

89 Ghashghaei H T, Weber J, Pevny L, et al. The role of neuregulinErbB4 interactions on the proliferation and organization of cells in the subventricular zone. Proc Natl Acad Sci USA, 2006, 103: 19301935

90 Meyer U, Feldon J. Epidemiology-driven neurodevelopmental animal models of schizophrenia. Prog Neurobiol, 2010, 90: 285-326

91 Anderson G, Maes M. Schizophrenia: Linking prenatal infection to cytokines, the tryptophan catabolite (TRYCAT) pathway, NMDA receptor hypofunction, neurodevelopment and neuroprogression. Prog Neuropsychopharmacol Biol Psychiatry, 2012, 42: 5-19

92 Meyer U, Knuesel I, Nyffeler M, et al. Chronic clozapine treatment improves prenatal infection-induced working memory deficits without influencing adult hippocampal neurogenesis. Psychopharmacology (Berl), 2010, 208: 531-543

93 Toblin R L, Riviere L A, Thomas J L, et al. Grief and physical health outcomes in US soldiers returning from combat. J Affect Disord, 2012, 136: 469-475

94 Dayer A G, Cleaver K M, Abouantoun T, et al. New GABAergic interneurons in the adult neocortex and striatum are generated from different precursors. J Cell Biol, 2005, 168: 415-427

95 Fowler C D, Liu Y, Wang Z. Estrogen and adult neurogenesis in the amygdala and hypothalamus. Brain Res Rev, 2008, 57: 342-351

96 Lieberwirth C, Liu Y, Jia X, et al. Social isolation impairs adult neurogenesis in the limbic system and alters behaviors in female prairie voles. Horm Behav, 2012, 62: 357-366

97 Okuda H, Tatsumi K, Makinodan M, et al. Environmental enrichment stimulates progenitor cell proliferation in the amygdala. $\mathrm{J}$ Neurosci Res, 2009, 87: 3546-3553

98 Ferguson J N, Young L J, Insel T R. The neuroendocrine basis of social recognition. Front Neuroendocrinol, 2002, 23: 200-224

99 Mercadante M T, Cysneiros R M, Schwartzman J S, et al. Neurogenesis in the amygdala: A new etiologic hypothesis of autism? Medical hypotheses, 2008, 70: 352-357

100 Wang Y, Fontanini A, Katz D B. Temporary basolateral amygdala lesions disrupt acquisition of socially transmitted food preferences in rats. Learn Mem, 2006, 13: 794-800

101 Silverman J L, Smith D G, Rizzo S J, et al. Negative allosteric modulation of the mGluR5 receptor reduces repetitive behaviors and rescues social deficits in mouse models of autism. Sci Transl Med, 2012, 4: 131-151

102 Munson J, Dawson G, Abbott R, et al. Amygdalar volume and behavioral development in autism. Arch Gen Psychiatry, 2006, 63: 686-693

103 Howard M A, Cowell P E, Boucher J, et al. Convergent neuroanatomical and behavioural evidence of an amygdala hypothesis of autism. Neuroreport, 2000, 11: 2931-2935

104 Gould E, Reeves A J, Graziano M S, et al. Neurogenesis in the neocortex of adult primates. Science, 1999, 286: 548-552

105 Arvidsson A, Collin T, Kirik D, et al. Neuronal replacement from endogenous precursors in the adult brain after stroke. Nat Med, 2002, 8: 963-970

106 Leker R R, Soldner F, Velasco I, et al. Long-lasting regeneration after ischemia in the cerebral cortex. Stroke, 2007, 38: 153-161

107 Ohira K, Furuta T, Hioki H, et al. Ischemia-induced neurogenesis of neocortical layer 1 progenitor cells. Nat Neurosci, 2010, 13: 173179

108 Kornack D R, Rakic P. Cell proliferation without neurogenesis in adult primate neocortex. Science, 2001, 294: 2127-2130

109 Banasr M, Duman R S. Glial loss in the prefrontal cortex is sufficient to induce depressive-like behaviors. Biol Psychiatry, 2008, 64: $863-870$

110 Czeh B, Perez-Cruz C, Fuchs E, et al. Chronic stress-induced cellular changes in the medial prefrontal cortex and their potential clinical implications: Does hemisphere location matter? Behav Brain Res, 2008, 190: 1-13

111 Cotter D R, Pariante C M, Everall I P. Glial cell abnormalities in major psychiatric disorders: The evidence and implications. Brain Res Bull, 2001, 55: 585-595

Open Access This article is distributed under the terms of the Creative Commons Attribution License which permits any use, distribution, and reproduction in any medium, provided the original author(s) and source are credited. 